\title{
Observations of Beam Losses Due to Bound-Free Pair Production in a Heavy-Ion Collider
}

\author{
R. Bruce, ${ }^{*}$ J. M. Jowett, and S. Gilardoni \\ CERN, Geneva, Switzerland \\ A. Drees, W. Fischer, and S. Tepikian \\ $B N L$, Upton, New York, USA \\ S. R. Klein \\ LBNL, Berkeley, California, USA
}

(Received 13 June 2007; published 3 October 2007)

\begin{abstract}
We report the first observations of beam losses due to bound-free pair production at the interaction point of a heavy-ion collider. This process is expected to be a major luminosity limit for the CERN Large Hadron Collider when it operates with ${ }^{208} \mathrm{~Pb}^{82+}$ ions because the localized energy deposition by the lost ions may quench superconducting magnet coils. Measurements were performed at the BNL Relativistic Heavy Ion Collider (RHIC) during operation with $100 \mathrm{GeV} /$ nucleon ${ }^{63} \mathrm{Cu}^{29+}$ ions. At RHIC, the rate, energy and magnetic field are low enough so that magnet quenching is not an issue. The hadronic showers produced when the single-electron ions struck the RHIC beam pipe were observed using an array of photodiodes. The measurement confirms the order of magnitude of the theoretical cross section previously calculated by others.
\end{abstract}

DOI: 10.1103/PhysRevLett.99.144801

PACS numbers: 29.20.Dh, 25.75. $-\mathrm{q}$

When fully-stripped heavy ions of atomic numbers $Z_{1}$, $Z_{2}$ are brought into collision at the interaction point (IP) of a collider, a number of electromagnetic interactions are induced by the intense fields generated by the coherent action of all the $Z_{1,2}$ charges in either nucleus (for a review, see Ref. [1]). Some of these "ultraperipheral" interactions have much higher cross sections than the hadronic nuclear interactions that are the main object of study. Among them, the bound-free pair production (BFPP), sometimes known as electron capture from pair production, occurs when the virtual photon exchanged by the ions converts into a pair, and the electron is created in an atomic shell of one of the ions:

$$
Z_{1}+Z_{2} \stackrel{\gamma}{\rightarrow}\left(Z_{1}+e^{-}\right)_{1 s_{1 / 2}, \ldots}+Z_{2}+e^{+}
$$

The resulting one-electron atoms have a slightly larger magnetic rigidity than the original bare nucleus. (Magnetic rigidity is defined as $p /(Q e)=B \rho$ for a particle with momentum $p$ and charge $Q e$ that would have bending radius $\rho$ in a magnetic field $B$ ). Since the transverse recoil is very small, this "secondary beam" will emerge at a very small angle to the main beam. However, it will be bent and focused less by the guiding magnetic elements and may be lost somewhere in the collider ring.

It has long been known that this process, together with electromagnetic dissociation of the nuclei, could be a major contribution to the intensity and luminosity decay of ion colliders [2,3]. It was realized more recently [4,5] that, in certain conditions, the BFPP beam will be lost in a well-defined spot, initiating hadronic showers in the vacuum envelope of the beam. The resulting localized heat deposition could induce quenches of superconducting magnets. Detailed calculations have been given elsewhere for $2.76 \mathrm{TeV} /$ nucleon ${ }^{208} \mathrm{~Pb}^{82+}$ operation of the $\mathrm{LHC}$ at CERN [5-8]. The consequent luminosity limit is expected to occur at a level close to the design performance. It is therefore vital to test our quantitative understanding of the features of the BFPP process in order to ensure safe operation of the LHC, uninterrupted by lengthy quenchrecovery procedures. BFPP has been measured in fixed target experiments [9-11] at lower energy but not, until now, in a collider. An opportunity arose during ${ }^{63} \mathrm{Cu}^{29+}$ operation of RHIC at Brookhaven National Laboratory.

The flux of ions in the secondary BFPP beam emerging from the interaction point is the product of the collider luminosity and the BFPP cross section. The partial cross section for electron capture for a given low-lying atomic bound state $i$ on nucleus 1 has the approximate form [12]

$$
\sigma_{i} \approx Z_{1}^{5} Z_{2}^{2}\left(A_{i} \log \gamma_{\mathrm{cm}}+B_{i}\right)
$$

where $\gamma_{\mathrm{cm}}$ is the Lorentz boost of the ions in the center-ofmass frame and $A_{i}, B_{i}$ depend only weakly on $Z$. The total cross section for BFPP to one of the colliding ions is then

$$
\sigma_{\mathrm{BFPP}}=\sum_{i} \sigma_{i}
$$

where the sum is over all atomic shells. Some numerical values from [12] are given in Table I. The cross section for collisions of $100 \mathrm{GeV} /$ nucleon ${ }^{63} \mathrm{Cu}^{29+}$ was not calculated in Ref. [12] but we have estimated it as $0.2 \mathrm{~b}$ by interpolation of the data given in Fig. 7 of Ref. [12], scaled with $Z$ and $\log \gamma_{\mathrm{cm}}$ according to Eq. (2). Applied to other tabulated values in Ref. [12], this method produces an agreement within $10 \%$. 
TABLE I. BFPP cross sections, typical peak luminosity, BFPP rates and relative changes in magnetic rigidity at RHIC and LHC. Values are taken directly where possible, or estimated by fitting sums of contributions of the form (2), to the information in Ref. [12]. $\delta=1 /(Z-1)$ is the fractional deviation of the magnetic rigidity.

\begin{tabular}{lcccc}
\hline \hline & $\sigma_{\text {BFPP }}$ (barn) & $L / 10^{27}\left(\mathrm{~cm}^{-2} \mathrm{~s}^{-1}\right)$ & BFPP rate $(\mathrm{kHz})$ & $\delta(\%)$ \\
\hline LHC Pb-Pb 2759 GeV/nucleon & 281 & 1 & 281 & 1.2 \\
RHIC Au-Au 100 GeV/nucleon & 114 & 3 & 342 & 1.3 \\
RHIC Cu-Cu 100 GeV/nucleon & 0.2 & 20 & 4 & 3.6 \\
RHIC Cu-Cu 31 GeV/nucleon & 0.08 & 1 & 0.08 & 3.6 \\
\hline \hline
\end{tabular}

The impact point of the lost ions is predicted by tracking the orbit of the BFPP beam in the collider optics until it intersects the physical aperture (vacuum pipe) of the beam line. To lowest order in the uncorrelated betatron amplitudes, $a, b$, and relative magnetic rigidity deviation from the central value, $\delta$, the horizontal displacement from the central orbit, at a distance $s$ from the IP is

$$
x(s)=a \sqrt{2 \beta(s)} \cos [\mu(s)]+b \sqrt{2 \beta(s)} \sin [\mu(s)]+d_{x}(s) \delta .
$$

Averaging over all ions gives the transverse emittance of the beam: $\left\langle a^{2}+b^{2}\right\rangle=\epsilon ; \beta(s)$ is the usual "Twiss function" determined by the focusing properties of the accelerator, $\mu(s)=\int_{0}^{s} \beta(u)^{-1} d u$ is the betatron phase and $d_{x}(s)$ is the locally generated dispersion $\left(d_{x}(0)=d_{x}^{\prime}(0)=0\right)$ that encodes the spectrometer effect of the bending magnets.

An ion following the central trajectory (i.e., in the middle of the bunch) in the BFPP beam enters the IP with $a=b=\delta=0$ and emerges, still with $a=b=0$, but $\delta=1 /(Z-1)$. This ion will hit the beam pipe at the first location where the horizontal aperture $A_{x}$ satisfies $A_{x}(s)=d_{x}(s) /(Z-1)$. In the arc, $A_{x}=3.455 \mathrm{~cm}$.

During ${ }^{197} \mathrm{Au}^{79+}$ operation at RHIC, $\delta$ is not large enough for this to occur $\left(x_{\max } \simeq 2.5 \mathrm{~cm}\right)$. However, during ${ }^{63} \mathrm{Cu}^{29+}$ runs [13], the larger $\delta$ (Table I) meant that the ions should be lost in a well-defined location where they can be detected directly. Thanks to the small cross section, there was no risk of quenching any superconducting materials. Since the BFPP beam was not visible to the existing detectors, beam loss monitors in the form of $p-i-n$ diodes (PDs) of the type Hamamatsu S3590 were mounted on the outside of the magnet cryostat downstream of the IP of the PHENIX experiment. The PDs have a time resolution of $25 \mathrm{~ns}$ and the counts were read out every second. They were initially positioned in a wide configuration (WPD), $3 \mathrm{~m}$ apart (see Fig. 1), and later moved closer together (CPD), $0.5 \mathrm{~m}$ apart, around an observed count-rate maximum at $141.6 \mathrm{~m}$.

A linear interpolation of $d_{x}(s)$ between magnet ends, based on the design model of the collider, identified the elements within which the BFPP beam was lost: a $9.7 \mathrm{~m}$ long dipole magnet starting at $s=129.6 \mathrm{~m}$, followed by a $2.12 \mathrm{~m}$ drift space and a quadrupole magnet, shown in Fig. 1. More precise tracking, including chromatic effects ( $\delta$ dependences), and a final step of analytic orbit calcula- tion inside the dipole, predicted an impact at $s_{i} \simeq 135.5 \mathrm{~m}$ with an angle of incidence of $\left\langle x^{\prime}\left(s_{i}\right)\right\rangle \simeq 2.7 \mathrm{mrad}$. Tracking an assumed Gaussian distribution of the amplitudes $a$ and $b$ in Eq. (4) gives the distribution of impact momenta in the spot around this point. Figure 2 shows the main ${ }^{63} \mathrm{Cu}^{29+}$ beam together with the ${ }^{63} \mathrm{Cu}^{28+}$ BFPP beam propagating from the IP.

To the extent that the RHIC hardware differs from the design model, the impact point may be shifted. The global distortion of the central orbit by misalignments of the magnets is partially corrected by localized corrector magnets. A least-squares fit was made to orbit data from beam position monitors (BPMs) and measured misalignments (of order 1-2 $\mathrm{mm}$ ) of the quadrupole magnets in the form

$$
\begin{aligned}
x(s)= & x_{\beta}(s)+\sum_{j} \Theta\left(s-s_{j}\right) \\
& \times \sqrt{\beta(s) \beta\left(s_{j}\right)} \sin \left[\mu(s)-\mu\left(s_{j}\right)\right] \theta_{j} .
\end{aligned}
$$

Here $\Theta(s)$ is the unit step function, $x_{\beta}$ the first two terms in Eq. (4) and $\theta_{j}$ are the angular kicks from misalignments and correctors at locations $s_{j}$. Unfortunately not enough data were available to make a satisfactory fit. Instead, the possible spread of the impact point was estimated by calculating the propagated error using Eq. (5). A possible error on the horizontal quadrupole misalignments of $1 \mathrm{~mm}$ was assumed, corresponding to the $95 \%$ confidence inter-

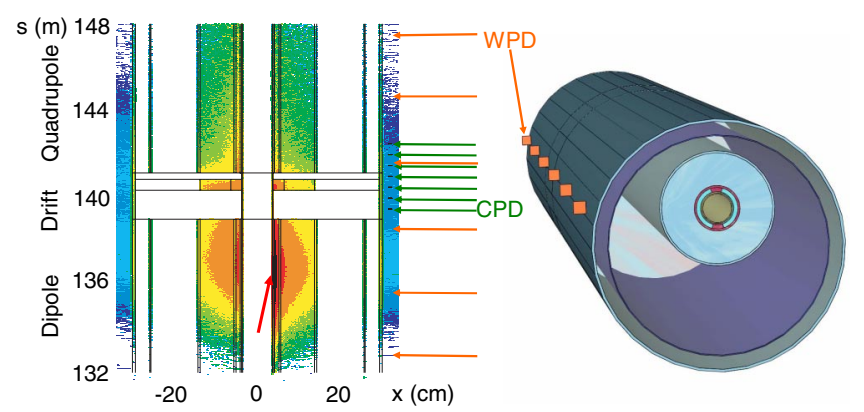

FIG. 1 (color online). Left: The energy deposition from a typical shower shown in a thin slice in the $x$-s plane through the geometry. The arrow (red online) near the center of the figure indicates the impact point and the arrows along the right side (green and orange online) show the positions of the PDs in the CPD and WPD. Right: The 3D model of the geometry as implemented in FLUKA around the impact point. The squares (orange online) show the PDs in the WPD. 
$\mathrm{x}(\mathrm{m})$

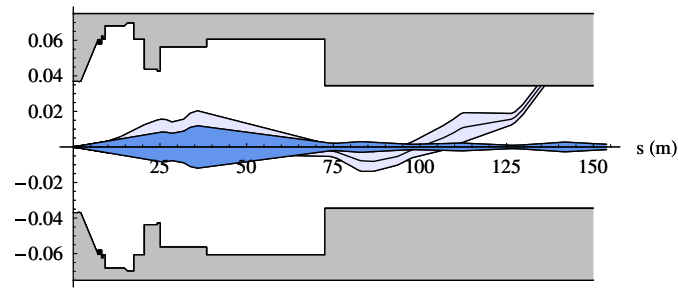

FIG. 2 (color online). The horizontal projection of the $1 \sigma$ envelope of the BFPP beam and the nominal beam emerging from the PHENIX IP. The BFPP beam hits the inside of the vacuum chamber at $135.5 \mathrm{~m}$.

val of the measurement and the fact that the magnets may have moved between early 2005 when the BFPP measurement was done and late 2006 when the misalignments were measured. The absolute error on the BPM data was taken to be $0.5 \mathrm{~mm}$, reflecting the limited data and possible nonreproducibility. Combining these errors gives an impact point of $135.5 \pm 2 \mathrm{~m}$. Keeping the nominal optics, but displacing the beam pipe by $1.4 \mathrm{~mm}$ at the impact point according to the measured misalignment of the dipole, moves the impact point to $136.0 \mathrm{~m}$.

Several distributions of BFPP ions at the PHENIX IP, centered on different initial offsets and angles, were tracked to obtain their impact coordinates on the inside of the vacuum pipe. These were fed into a 3D model (see Fig. 1) of the impact region geometry, the PDs, and the dipole magnetic field, implemented in the FLUKA 2006.3 Monte Carlo code [14-16], in order to simulate the shower in the magnet and particles emerging from the cryostat. This code has been benchmarked by others in the relevant energy range [16].

Within 2-2.5 $\mathrm{m}$ from the average impact point most of the shower inside the magnet has died out, although particles outside propagate farther. This can be understood through a rough estimate: The typical average nuclear interaction length for the superconducting coil and the iron cold bore is about $20 \mathrm{~cm}$. Convoluted with the impact point spot size of about $1.8 \mathrm{~m}$, this gives a total shower length of about $2.8 \mathrm{~m}$, assuming that the shower lasts five interaction lengths. The maximum PD signal was expected about $2.5 \mathrm{~m}$ downstream of the impact point. When the shower is contained within the dipole, moving the impact point along $s$ only translates the shower. If the impact point is moved closer toward the end of the magnet or the angle of incidence decreased, more of the shower emerges into the void before the quadrupole and the profile changes qualitatively. A second peak in energy deposition appears at the entrance of the quadrupole and eventually exceeds the first one.

The PDs count the number of minimum-ionizing particles (MIPs) that pass their active area of $1 \mathrm{~cm}^{2}$. In the simulation, the number of MIPs entering each PD was recorded, assuming a detection efficiency of $30 \%$. Since the PDs are much more sensitive to particles entering through the side orthogonal to the cryostat, only these particles were counted. At the expected BFPP ion production rate of $4 \mathrm{kHz}$, and the 0.01 MIPs expected per PD per ion, pileup from multiple interactions was not an issue. Both WPD and CPD configurations were simulated for various impact points and a typical result is shown together with measured data in Fig. 3.

Data were collected from the PDs during 14 fills of RHIC with ${ }^{63} \mathrm{Cu}^{29+}$ ions. An example of the recorded signal is shown in Fig. 4, together with the zero degree calorimeter (ZDC) count rate (which is directly proportional to the luminosity). The PD signals show a good temporal correlation with luminosity and are very localized along $s$, close to the predicted impact position. With a $1 \mathrm{~s}$ binning, the correlation coefficient, $r$, between the PD signal and ZDC is 0.7 at $141.6 \mathrm{~m}$ and decreases gradually to 0.1 at PDs outside the predicted impact area. With a $30 \mathrm{~s}$ binning the highest $r$ rises to 0.98 . This makes it very unlikely that some other source than the collisions could be responsible for the signals.

This becomes even clearer from van der Meer scans in which the beam orbits are scanned transversely across each other and the luminosity variation is recorded as in Ref. [17]. Figure 5 shows the ZDC rate and the signal of one of the PDs as a function of the orbit bump amplitude.

Since the data were taken parasitically during normal colliding-beam operation, the PD signals were often polluted by other losses, e.g., from collimation. Therefore the cleanest data sets with least interference were picked out (near the beginning of each fill) and the averaged count rate with background subtracted, normalized to luminosity, was calculated for each PD (see Fig. 3). The background noise level was calculated for each PD as the average signal without luminosity.

In the WPD, the maximum signal was recorded by the $\mathrm{PD}$ at $141.6 \mathrm{~m}$ but moved to $140.5 \mathrm{~m}$ in the CPD. The count rates ranged from 1 to $20 \mathrm{~Hz}$ depending on PD position and

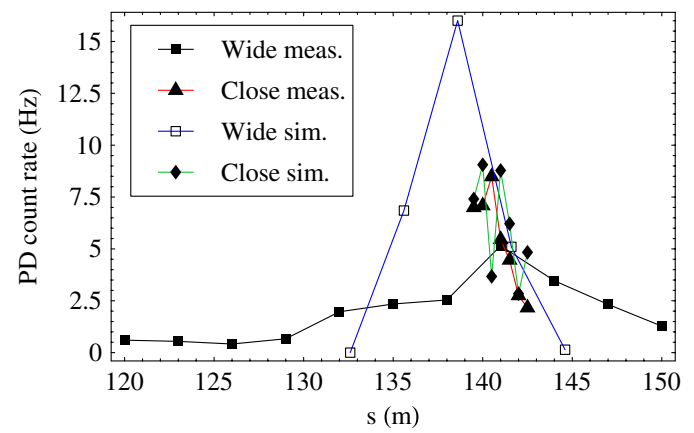

FIG. 3 (color online). The PD signals from both configurations together with simulation results, averaged over the fills and normalized to a typical average luminosity of $9.1 \times 10^{27} \mathrm{~cm}^{-2} \mathrm{~s}^{-1}$. The simulation was produced from the central BFPP orbit in the nominal lattice but with the beam pipe misaligned by $1.4 \mathrm{~mm}$ at the impact location according to measurements. Because of large statistical error bars, the simulation gives only the order of magnitude to expect in the measurement. 


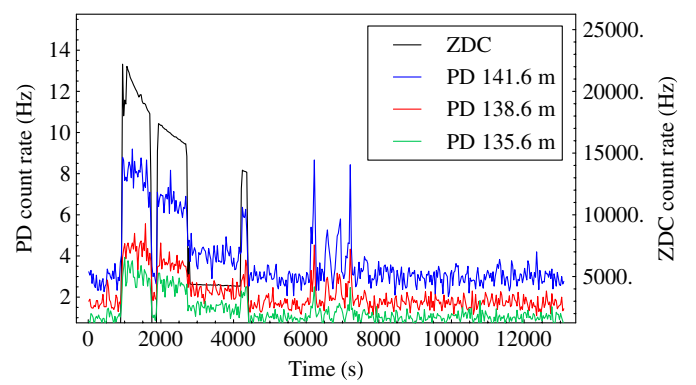

FIG. 4 (color online). Count rates measured on the ZDC luminosity monitors (black, right scale) and the three PDs with the highest signal [shades of gray, left scale (colors online)] during a store with the WPD. The data was binned in $30 \mathrm{sec}$ intervals. A clear correlation between the luminosity and the PD count rates can be seen.

luminosity. This agrees well with the simulation. However, the maximum in the simulation came from the PD at $138.6 \mathrm{~m}$, which does not exactly agree with the measurements. This means that, in reality, the second peak in the energy distribution outside the cryostat is actually higher than the first, while in the simulation the first peak is higher. As can be seen in Fig. 1, the peak in the shower could also escape between the PDs in the WPD. However, if the impact point is translated within the $2 \mathrm{~m}$ error bar, the simulated maximum moves further downstream and a fair agreement can be found. Moreover, the PD signals themselves have error bars-apart from the statistical error a small relative change in the counting efficiency between the PDs could change the result. This has not been measured so we give no numerical estimate.

One might hope to extract a value for the cross section for BFPP between colliding copper ions at $100 \mathrm{GeV} /$ nucleon. However, because of the uncertainties in both the number of MIPs entering a specific diode in the simulation and the recorded count rates, we can only conclude from the good agreement that the theoretical estimate of the BFPP cross section has the right order of magnitude.
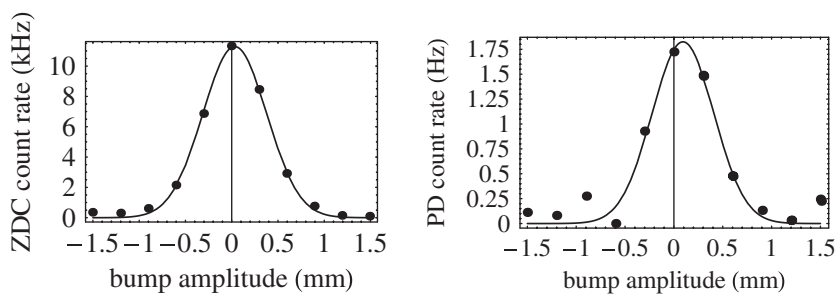

FIG. 5. The ZDC count rate (proportional to luminosity, left) and background-subtracted PD count rate (right) vs relative orbit displacement between beams in a van der Meer scan. A Gaussian fit of the form $A \exp \left(\frac{-\left(x-x_{0}\right)^{2}}{\sigma_{x}^{2}}\right)$ is shown together with the data. The two fits had $\sigma_{x}=0.49 \mathrm{~mm}$ and $0.44 \mathrm{~mm}$ and $\chi^{2} / \mathrm{DOF}=$ 0.23 and 0.20 , assuming a measurement error of $500 \mathrm{~Hz}$ on the ZDC signal and $0.3 \mathrm{~Hz}$ on the PD.
No signal was detectable at $31 \mathrm{GeV} /$ nucleon, consistent with the much lower $\left(L \times \sigma_{\mathrm{BFPP}}\right)$ (see Table I).

In conclusion, we made the first measurements of the localized loss of a BFPP generated secondary beam in an ion collider. These measurements were done with copper beams at RHIC. We found the location of the maximum loss monitor signal at $140.5 \mathrm{~m}$ from the IP, within $2 \mathrm{~m}$ of its calculated location, and the measured event rates in our PD detectors within a factor of 2 of calculated ones. The deviations between calculated and measured values are consistent with estimated errors. This is a valuable test of our ability to make quantitative predictions of this effect for the LHC, where it is expected to be one of the most restrictive luminosity limits.

We thank A.J. Baltz, R. Gupta, K. Hencken, J. B. Jeanneret and T. Roser for helpful discussions.

*Also at MAXlab, Lund University, Sweden.

Electronic address: roderik.bruce@cern.ch

[1] C. Bertulani, S. R. Klein, and J. Nystrand, Annu. Rev. Nucl. Part. Sci. 55, 271 (2005).

[2] H. Gould, Lawrence Berkeley Laboratory Report No. LBL-18593, 1984.

[3] A. J. Baltz, M. J. Rhoades-Brown, and J. Weneser, Phys. Rev. E 54, 4233 (1996).

[4] S. R. Klein, Nucl. Instrum. Methods Phys. Res., Sect. A 459, 51 (2001).

[5] J. M. Jowett, J.-B. Jeanneret, and K. Schindl, Proceedings of the Particle Accelerator Conference 2003, Portland, Oregon, edited by Joe Chew, Peter Lucas, and Sara Webber (IEEE, Piscataway, New Jersey, 2003), p. 1682.

[6] O.S. Brüning et al., CERN Report No. CERN-2004-003V1, 2004.

[7] J. M. Jowett, R. Bruce, and S. Gilardoni, Proceedings of the Particle Accelerator Conference 2005, Knoxville, Tennessee (IEEE, Piscataway, New Jersey, 2005), p. 1306.

[8] R. Bruce, S. Gilardoni, and J. M. Jowett, CERN LHC Project Note 379, 2006.

[9] A. Belkacem et al., Phys. Rev. Lett. 71, 1514 (1993).

[10] H. F. Krause et al., Phys. Rev. Lett. 80, 1190 (1998).

[11] P. Grafström et al., Proceedings of the Particle Accelerator Conference 1999, New York (IEEE, Piscataway, New Jersey, 1999), p. 1671.

[12] H. Meier et al., Phys. Rev. A 63, 032713 (2001).

[13] F. Pilat et al., Proceedings of the Particle Accelerator Conference 2005, Knoxville, Tennessee (IEEE, Piscataway, New Jersey, 2005), p. 4281.

[14] A. Fasso et al., CERN Report No. CERN-2005-10, 2005.

[15] A. Fasso et al., Proceedings of the Computing in High Energy and Nuclear Physics 2003 Conference, La Jolla, California (SLAC, Stanford, California, 2003).

[16] S. Roesler, R. Engel, and J. Ranft, SLAC Report No. SLAC-PUB-8740, 2000.

[17] A. Drees et al., Proceedings of the Particle Accelerator Conference 2003, Portland, Oregon, edited by Joe Chew, Peter Lucas, and Sara Webber (IEEE, Piscataway, New Jersey, 2003), p. 1688. 